Bird Study

\title{
Anti-predator responses in the Chilean Swallow Tachycineta leucopyga breeding in northwest Patagonia
}

\section{Melina Barrionuevo, Valeria Ojeda, Natalie Dudinszky \& Silvina Ippi}

To cite this article: Melina Barrionuevo, Valeria Ojeda, Natalie Dudinszky \& Silvina Ippi (2019): Anti-predator responses in the Chilean Swallow Tachycineta leucopyga breeding in northwest Patagonia, Bird Study, DOI: 10.1080/00063657.2019.1617235

To link to this article: https://doi.org/10.1080/00063657.2019.1617235

曲 Published online: 31 May 2019.

Submit your article to this journal $\pi$

View Crossmark data $₫$ 


\title{
Anti-predator responses in the Chilean Swallow Tachycineta leucopyga breeding in northwest Patagonia
}

\author{
Melina Barrionuevo ${ }^{a}$, Valeria Ojeda ${ }^{a}$, Natalie Dudinszky ${ }^{a}$ and Silvina Ippi ${ }^{b}$

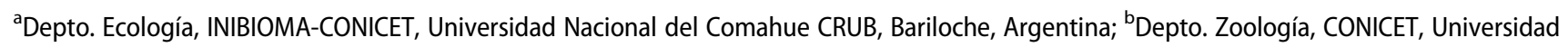 \\ Nacional del Comahue CRUB, Bariloche, Argentina
}

ABSTRACT

Capsule: Breeding Chilean Swallows Tachycineta leucopyga in Argentina use visual and acoustic signals in response to potential threats from a predator, but these did not vary with brood age.

Aims: To study anti-predator behavioural responses in breeding Chilean Swallows from North Patagonia, Argentina, by using a nest-box experiment.

Methods: At days eight and 14 after the first nestlings hatched, Chilean Swallow breeding adults were exposed to a stuffed decoys of either a natural local predator, the Austral Pygmy-Owl Glaucidium nana, or a neutral decoy, a stuffed Rufous-Collared Sparrow Zonotrichia capensis.

Results: Anti-predator behavioural responses consisted of displaying dives, circling flights and group mobbing around the predator decoy, together with the emission of several vocalizations, among which a mobbing alarm call was the most frequent. We did not find significant differences in the intensity of parental nest defence behaviours at different nestling ages ( 8 and 14 days old).

Conclusion: Anti-predator behaviour of breeding Chilean Swallows consists of visual and acoustic signals, involving swallows other than the nest owners, and without variation during the nestling period. Our exploratory assessment on the anti-predator behaviour of Chilean Swallows is the first description of the southernmost Neotropical swallow defence behaviour and will therefore help delineate future research.
ARTICLE HISTORY

Received 9 August 2018

Accepted 1 February 2019
Predation has long been recognized as a major demographic driver in most wild species. In birds, it is the most important cause of nest failure (Ricklefs 1969). Individuals displaying more nest defence behaviours have been found to have a lower probability of predation of eggs, nestlings or fledglings, which leads to increased breeding success (Greig-Smith 1980, Vrublevska et al. 2015). However, nest defence can also increase parental costs because it increases (1) their risk of predation and (2) the energy and time allocated to defensive behaviours, which are subtracted from other activities such as foraging (Montgomerie \& Weatherhead 1988) or attending the nest (Zanette et al. 2011).

Parental defence may vary according to several factors, for example, clutch size (Svagelj et al. 2012), type of nest predator (Mahr et al. 2015), and parent sex (Hogstad 2005) and personality (Kontiainen et al. 2009). Also, nest defence has been shown to increase with nestling age (Montgomerie \& Weatherhead 1988, Redondo \& Carranza 1989, Shew et al. 2016), which has been attributed to several non-mutually exclusive hypotheses. For example, as nestlings age, they become more conspicuous and detectable by predators (Harvey \& Greenwood 1978, Redondo \& Carranza 1989). Moreover, the reproductive value of the brood increases because the probability of re-nesting decreases as the season progresses (Barash 1975, Redondo \& Carranza 1989), which may lead to greater nest defence.

Swallows of the genus Tachycineta were studied for decades through the 'Golondrinas de las Américas' program, with standardized protocols. Research has shown that northern and southern Hemisphere species have different life-history traits. Southern species have smaller clutches, lower nestling growth rates, and longer nestling periods than northern species (Massoni et al. 2007, Liljesthrom 2011, Ospina et al. 2015). While anti-predatory behaviours are largely known for northern congeners (Winkler 1991, 1992), studies on the southern species are recent and scant (Wischhoff et al. 2018).

Our aim was to explore the defence behaviour of breeding Chilean Swallows Tachycineta leucopyga, one of the Hirundinidae with the most southerly distribution, which breeds in the Patagonian Andes of 
Argentina and Chile during the austral summer. Based on what is known about the life-history of this and other Tachycineta species, we predicted that (a) breeding Chilean Swallows would perform antipredator responses against a predator decoy relative to a non-predatory control, and (b) their responses would be stronger as nestlings aged.

\section{Methods}

\section{Study site}

The study was carried out at two livestock ranches located near Bariloche city (northwest Argentine Patagonia): Fortín Chacabuco $\left(41^{\circ} 00^{\prime} \mathrm{S} 71^{\circ} 10^{\prime} \mathrm{W}\right)$ and El Desafío $\left(41^{\circ} 15^{\prime} \mathrm{S} 71^{\circ} 10^{\prime} \mathrm{W}\right)$, which are $30 \mathrm{~km}$ distant from each other. Both ranches were located within the steppeforest ecotone, and crossed by small streams (Chacabuco and La Mina, respectively) that are particularly attractive to swallows. The regional climate was cold-temperate with a mean annual temperature of $8.6^{\circ} \mathrm{C}$. Mean annual precipitation was about $580 \mathrm{~mm}$, mostly occurring during the winter months. Strong westerly winds blew throughout the year but predominantly during the summer months (Paruelo et al. 1998).

\section{Study species}

Like all the members of the Tachycineta genus, Chilean Swallows are migratory, secondary cavity nesters that naturally nest in trees (Johnson 1967). They are socially monogamous aerial insectivores with biparental care (Liljesthrom 2011).

\section{Field methods}

From November to February 2017, we studied Chilean Swallows breeding in nest-boxes. Nest-boxes were built according to Liljesthrom (2011) and mounted at a height of $1.4-1.7 \mathrm{~m}$ on fenceposts already present on the ranches. Each box was placed at a distance of approximately $30 \mathrm{~m}$ from the next (40 boxes at El Desafío and 15 at Fortín Chacabuco). Laying dates were determined by daily monitoring of the nest-boxes from mid-November until the clutch was complete. Afterwards, nest-boxes were checked every two days, on average. Fifteen days after laying had finished, nests were visited daily to record hatching dates.

\section{Nest defence experiment}

We investigated anti-predator responses in 12 breeding pairs (two at Fortín Chacabuco and ten at El Desafío), by performing two sequential trials at each nest: at day eight (8-d trial), and at day 14 (14-d trial) after hatching of the first nestling. In each nest and for each age, we measured the adults' behavioural response to the presence of two stuffed decoys: an Austral PygmyOwl Glaucidium nana (owl model), as a predator, and a Rufous-Collared Sparrow Zonotrichia capensis (neutral model), as a control. The Austral Pygmy-Owl shows mainly diurnal activity, has a large proportion of non-rodent food in its diet and has been recorded attempting to prey on a Chilean Swallow nest, although unsuccessfully (Ibarra et al. 2014). The Rufous-Collared Sparrow is a widespread granivorous Neotropical passerine that builds open cup nests. At both the 8-d and the 14-d trials, the owl and neutral models were presented separated by a time interval of $24 \mathrm{~h}$, in random order. At two boxes at El Desafío, nestlings died before we could run the 14-d trials. Therefore, we performed a total of 22 neutral and owl model trials: 128 -d trials, and $1014-\mathrm{d}$ trials. The trials were conducted from $08: 00 \mathrm{~h}$ to $13: 00 \mathrm{~h}$ by mounting the models on wires attached to adjacent fences, 1$1.5 \mathrm{~m}$ away from the focal nest-box.

We registered the immediate response of the adults outside the nest-boxes to the presence of the stuffed decoy. Trials began when at least one swallow was present near its nest, and was therefore able to respond to the decoy stimulus. The observer recorded the behavioural responses from a spot 20-30 $\mathrm{m}$ from the nest-box, with a Sony Handycam CX405 digital camera. Each trial lasted three minutes during which the observer remained motionless and hidden among the vegetation. Meanwhile, we also recorded the vocalizations emitted by the swallows, with a Marantz Professional PMD660 sound digital recorder, equipped with a Senheiser ME 66 directional microphone, with a sampling rate of $48 \mathrm{kHz}$. The microphone was mounted on a tripod $0.5 \mathrm{~m}$ above the ground, and 2-4 $\mathrm{m}$ from the nest.

\section{Data analysis}

In other swallow species, nest defence is a combination of passive and active behaviours (Winkler 1994) and group mobbing is common (Shields 1984). Passive behaviour involves displaying circling flights $3-5 \mathrm{~m}$ above the predator, and active behaviour involves diving towards the predator (sudden changes of flight direction aimed at the stimulus) and emitting alarm calls (Winkler 1994). Therefore, we evaluated the following visual behavioural variables when analysing the video images: (1) number of swallows involved in the defence behaviours, (2) number of dives (from touching the decoy, to approaching to almost $60 \mathrm{~cm}$ 
above it), (3) number of circling flights around the decoy $(30-120 \mathrm{~cm})$, (4) time spent perching on the wire (seconds), (5) minimum distance between perching swallows and decoy (m), (6) time to appearance of more than three swallows (seconds), and (7) time that swallows were absent from the scene (i.e. time spent outside the observer's visual range, in seconds).

Spectrogram analyses of vocalizations were conducted with the RAVEN PRO 1.5 software (Cornell Bioacoustic Laboratory, Cornell, USA). We inspected the audio recordings from each trial by using the pre-set parameters of the spectrograms in the software (Window-Type: Hann, size: 512 samples, $3 \mathrm{~dB}$ filter bandwidth: $135 \mathrm{~Hz}$; time grid overlap: 50\%; hop size: 256 samples; discrete Fourier transform (DFT) size: 512 samples, and grid spacing: $93.8 \mathrm{~Hz}$ ). We identified four clearly different vocalization types (three calls and one song) emitted by the swallows during the trials. Also, we visually counted the number of calls or songs of each type of vocalization in the spectrogram, as appropriate, in order to obtain their frequency per trial. We were unable to identify the individual swallow performing each vocalization. Therefore, we extracted calls and songs representative of each vocalization type per nest. We selected the vocalizations based on their quality and whenever there was no overlapping with other vocalizations emitted at the same time. Therefore, the sample size was variable for each vocalization type. We measured the following bioacoustic variables: minimum frequency $(\mathrm{kHz})$, maximum frequency $(\mathrm{kHz})$, bandwidth (difference between minimum and maximum frequency, $\mathrm{kHz}$ ), peak frequency (defined as the frequency with maximum energy in the power spectrum of the call, $\mathrm{kHz}$ ), and duration of each call or song (seconds), depending on the type of signal.

To reduce the number of variables used in the analyses, we performed a Principal Component Analysis (PCA) with the following visual behavioural variables for each trial performed in each nest: $1,2,3$, 4 , and the frequency of the vocalizations named A, B, and $C$. The visual behaviour variables 5 and 6 were not included in the analysis because not all trials had swallows perching (5) and not in all trials did three or more swallows appear on scene (6). The frequency of the vocalization named $\mathrm{D}$, and the time that swallows were out of the observer's visual range (visual variable 7 ), were excluded from the PCA because of low factor loadings. We extracted two principal components (PCs) that were Varimax rotated (Kaiser 1958). We analysed the two PCs as dependent variables using a Linear Mixed Model, including the decoy (two level factor: owl or neutral model), hatchling age (two level factor: 8-d or 14-d), and their two-way interaction, as predictor variables. We included the nest as a random factor to account for the lack of independence between the trials run on the same nests (Zuur et al. 2009). We used a backwards selection procedure by removing the terms one by one (Crawley 2007), which followed a decreasing level of complexity (when interactions were present in the model), and a decreasing $P$ value. Then we compared the models with and without the eliminated variables with the 'ANOVA' function, using the Likelihood Ratio test (L-ratio). We used the package 'nlme' (Pinheiro et al. 2014) with the function 'Ime' of the R software version 2.12.1 (R Development Core Team 2010).

For the other statistical analyses including the PCA we used the Statistica v.7 (Statsoft Inc. 2004). Data are provided as mean \pm sd.

\section{Results}

\section{Description of defensive visual displays}

The typical behavioural response of Chilean Swallows in the presence of the owl model was to perform mobbing displays with many swallows that rapidly arrived on the scene, in contrast to the neutral model trials, where normally one or two swallows were present near the nest (Table 1).

During the owl model trials, the swallows approached the decoy, almost touching it, with diving and circling flights. Both variables were displayed significantly more often in owl model than in neutral model trials (Table 1; $n=22$; Wilcoxon: $z=3.77, P=0.0002 ; z=3.73$, $P=0.0002$, diving and circling flights respectively). As the swallows increased in numbers, higher numbers of

Table 1. Behavioural description of defensive anti-predator visual displays during model presentation trials conducted when Chilean Swallow nestlings were eight and 14 days old. When possible, mean \pm sd data is shown, also within brackets is shown the range of some variables. Variables from 1-4 were calculated for: $n=22$ trials for each decoy, variable 5: $n=22$ neutral model trials and $n=9$ owl model trials, variable $7: n=$ 1 neutral model trials and $n=13$ owl model trials.

\begin{tabular}{lcl}
\hline Variable & Neutral model trial & Owl model trial \\
\hline (1) Number of swallows & $1.68 \pm 0.57(1-3)$ & $3.27 \pm 0.42(1-7)$ \\
(2) Number of diving flights & $1.73 \pm 0.53(0-9)$ & $15.5 \pm 2.66(0-42)$ \\
(3) Number of circling flights & $3.64 \pm 2.85(0-10)$ & $12.0 \pm 4.65(3-15)$ \\
(4) Perching time (sec) & $96.5 \pm 13.7$ & $34 \pm 11.55$ \\
(5) Swallow-decoy distance & $2.80 \pm 2.37(0.3-10)$ & $4.06 \pm 2.21(2-7)$ \\
(m) & & \\
(6) Time with no swallows & $119.2 \pm 11.28(84-144)^{\mathrm{a}}$ & $100^{\mathrm{b}}$ \\
(sec) & \\
(7) Time to appearance of $>3$ & \\
$\quad$ swallows (sec) & \\
a $n=5$ trials, two of these cases occurred at the same nest on 8-d and 14-d \\
$\quad$ trials. \\
${ }^{b} n=1$ trial, this nest failed to raise the nestlings.
\end{tabular}




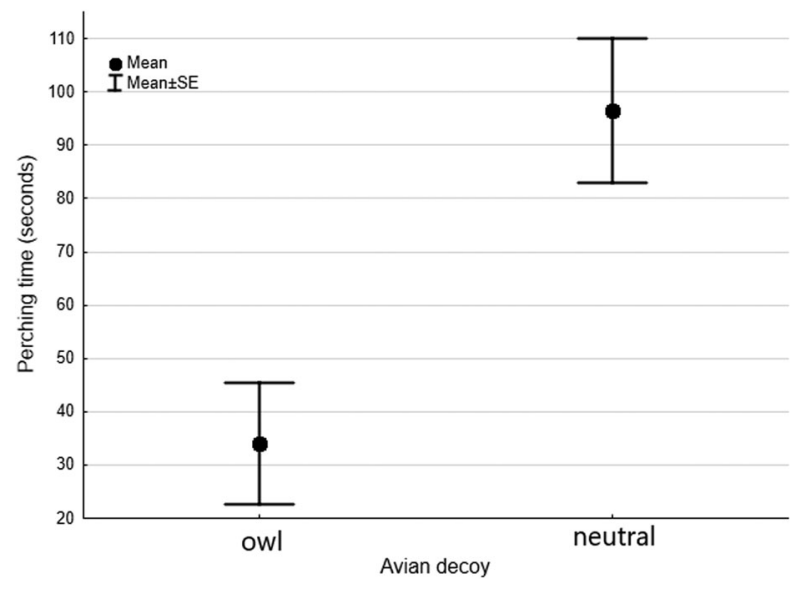

Figure 1. Time that Chilean Swallows spent perching on the wire of fences located near their nest-box in the presence of owl or neutral models, during three-minute trials. Dots and whiskers indicate mean time \pm se.

circling flights (correlation: $r=0.57, t=4.52, P<0.001$ ) and dives (correlation: $r=0.66, t=5.74, P<0.001$ ) were recorded. Also, circling flights and dives were positively related to each other (correlation: $r=0.71, t=6.52, P<$ 0.001).

During all the neutral model trials, the swallows remained perched on the wire during part of the trial, whereas in only nine owl model trials some swallows perched briefly, while other swallows continued attacking the decoy. Hence, the swallows perched for longer during the neutral model trials than during the owl model trials (Wilcoxon: $z=2.91, P=0.004, n=22$; Table 1 and Figure 1), but the average distance between the swallows and the decoy did not differ between neutral and owl model trials (Wilcoxon: $z=$ 1.82, $P=0.07, n=8$ ) (Table 1). In most of the trials, at least one swallow was present throughout the trial, with few exceptions (Table 1).

\section{Characterization of vocalizations}

We identified four different vocalizations (A-D, Table 2), and tentatively described their probable function. All were recorded at least once in both owl and neutral model trials (Table 3). The most frequent
Table 3. Presence (\%) of each vocalization type during owl and neutral model presentation trials conducted when Chilean Swallow nestlings were 8 and 14 days old.

\begin{tabular}{llcc}
\hline Vocalization & decoy type & day 8 & day 14 \\
\hline A & owl & 91.7 & 88.9 \\
& neutral & 66.7 & 90.0 \\
B & owl & 33.3 & 22.2 \\
& neutral & 8.3 & 10.0 \\
C & owl & 50.0 & 66.7 \\
& neutral & 41.7 & 50.0 \\
D & owl & 66.7 & 44.4 \\
& neutral & 75.0 & 80.0 \\
\hline
\end{tabular}

was vocalization A (Table 3), a high-intensity mobbing call which consisted of a short rasp sound, repeated at high frequency, mainly when mobbing the owl model (Figure 2, Table 4). Vocalization B was also a rasp call but with a broader bandwidth and a lower minimum frequency (Figure 2), emitted in several nests in both owl model and neutral model trials (Table 3), but not repeatedly (Table 4). Vocalization $\mathrm{C}$ was a short trill composed of only one syllable (Figure 2), and was present both in the owl and neutral model trials (Table 3). Vocalization D was a complex song with several variants, including different numbers and forms of syllables (Figure 2) (Table 3). We also recorded other vocalizations during the trials, but they occurred very rarely, or their recordings were of poor quality, precluding analysis.

\section{Nestling age and anti-predator responses}

Two PCs were extracted from the PCA, explaining 78.9\% of total variance (Table 5). Positive values of PC1 corresponded to an increasing number of dives, circling flights, swallows involved in defence behaviours, and A vocalizations; and were negatively related to the time spent perching (Table 5). Therefore, PC1 was interpreted as the active component of the anti-predator behaviour. The values of PC2 were highly and positively correlated with vocalizations B and $C$ (Table 5) but because the function of these vocalizations was unknown, we were unable to evaluate PC2 from a behavioural perspective. The interaction between the decoy (owl or neutral model) and the age

Table 2. Bioacoustic characterization of four different vocalizations ( $A, B, C$ and $D$ ) emitted by the Chilean Swallows in the surroundings of active nests during the experiments on anti-predator responses. Numbers between brackets indicate number of $n$ independent vocalizations each recorded in different nest-boxes (i.e. emitted in the surroundings of different nests) analysed. For each variable the mean $\pm s d$ is indicated.

\begin{tabular}{|c|c|c|c|c|c|}
\hline Vocalization & Minimum frequency $(\mathrm{kHz})$ & Maximum frequency $(\mathrm{kHz})$ & Band width (kHz) & Peak frequency $(\mathrm{kHz})$ & Duration (seconds) \\
\hline $\mathrm{A}(n=10)$ & $2.14 \pm 0.42$ & $17.50 \pm 3.73$ & $15.36 \pm 3.66$ & $5.22 \pm 0.40$ & $0.17 \pm 0.03$ \\
\hline $\mathrm{B}(n=4)$ & $0.69 \pm 0.34$ & $18.65 \pm 2.75$ & $17.68 \pm 3.16$ & $2.55 \pm 1.72$ & $0.22 \pm 0.07$ \\
\hline$C(n=8)$ & $1.77 \pm 0.31$ & $13.31 \pm 3.70$ & $11.53 \pm 3.61$ & $4.44 \pm 0.61$ & $0.13 \pm 0.02$ \\
\hline $\mathrm{D}(n=8)$ & $1.11 \pm 0.48$ & $12.39 \pm 4.65$ & $11.28 \pm 4.88$ & $4.15 \pm 0.70$ & $1.67 \pm 0.37$ \\
\hline
\end{tabular}


Table 4. Mean ( \pm se) frequency of calls (calls/min) emitted during decoy presentations when Chilean Swallow nestlings were 8 and 14 days old. Numbers in brackets indicate sample sizes.

\begin{tabular}{llcr}
\hline Vocalization & Decoy type & Day 8 (10) & Day 14 (10) \\
\hline A & owl & $44.92 \pm 7.97$ & $48.70 \pm 9.56$ \\
& neutral & $2.47 \pm 1.12$ & $9.40 \pm 1.89$ \\
B & owl & $1.14 \pm 0.99$ & $0.37 \pm 0.24$ \\
& neutral & $0.06 \pm 0.06$ & $0.13 \pm 0.13$ \\
C & owl & $3.11 \pm 1.54$ & $3.04 \pm 1.42$ \\
& neutral & $0.75 \pm 0.36$ & $0.53 \pm 0.22$ \\
D & owl & $2.81 \pm 0.85$ & $2.67 \pm 1.55$ \\
& neutral & $1.28 \pm 0.38$ & $0.67 \pm 0.24$ \\
\hline
\end{tabular}

of the first hatchling (8-d or 14-d) was not related to any of the PCA components (PC1: L-ratio $=0.88, P=0.35$; PC2: L-ratio $=0.05, P=0.82$ ). Moreover, behavioural responses expressed by the two PCs were similar at both times after hatching (Table 6). Nevertheless, PC1 (related to more active anti-predator behaviour) was significantly higher in the owl model trials than in the neutral model trials $(t=-6.27, P<0.001$; Figure 3$)$ but this was not the case for PC2 (Table 6).
Table 5. Variance for eigenvalues, and factor loadings of the first two PCA components for the seven variables considered in the analyses of anti-predator response experiments performed on Chilean Swallows.

\begin{tabular}{lcc}
\hline & PC1 & PC2 \\
\hline Eigenvalues & 4.15 & 1.37 \\
Total variance \% & 59.3 & 19.6 \\
Cumulative \% & 59.3 & 78.9 \\
Factor loadings & & \\
Dives & 0.93 & 0.46 \\
Circling & 0.91 & 0.11 \\
Perching & -0.82 & 0.13 \\
No. swallows & 0.76 & 0.43 \\
Vocalization A & 0.77 & 0.35 \\
Vocalization B & 0.18 & 0.84 \\
Vocalization C & 0.09 & 0.91 \\
\hline
\end{tabular}

\section{Discussion}

This is a first contribution to the understanding of antipredator behaviour in the southernmost breeding swallow. Defence behaviour of Chilean Swallow pairs breeding in North Patagonia consisted of dives, circling flights and increased vocal activity in association with
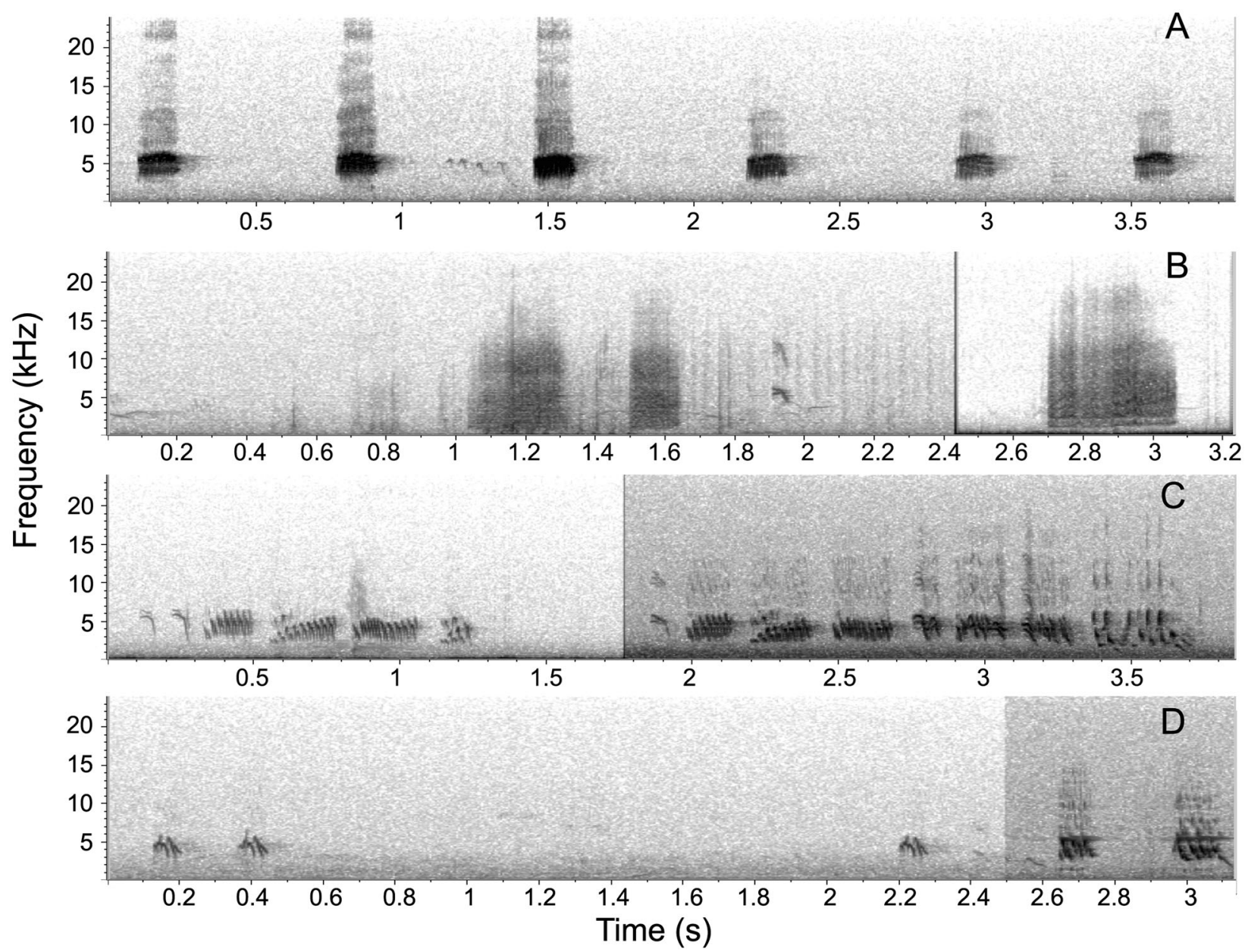

Figure 2. Spectrograms of the four vocalizations identified for the Chilean Swallows. Each capital letter in the figure (A, B, C and D) indicates a different vocalization type. With the exception of vocalizations $B$ and $C$, the rate of emission is representative of the natural rate. In figures $C$ and $D$, two variants of the same vocalization type are shown. 
Table 6. Results of the two Linear Mixed Models analyses to evaluate nest defence of Chilean Swallows in Northern Patagonia. Acoustic and visual variables were introduced in a PCA (Principal Component Analysis), and the first and second components were used as defence behaviour variables. Two different avian models (owl and neutral) were presented at different nestling ages (8 and 14 days; $n=10$ nests).

\begin{tabular}{llcc}
\hline Response variables & \multicolumn{1}{c}{ Fixed effects } & $\begin{array}{c}P \text { - } \\
\text { L-ratio }\end{array}$ & \begin{tabular}{c} 
value \\
\hline PC1
\end{tabular} \\
& Model (owl vs. neutral) & 27.3 & $<0.001$ \\
PC2 & First hatchling's age (8 vs. 14 days) & 1.19 & 0.27 \\
& Model (owl vs. neutral) & 1.50 & 0.22 \\
& First hatchling's age (8 vs. 14 days) & $<0.001$ & 0.99 \\
\hline
\end{tabular}

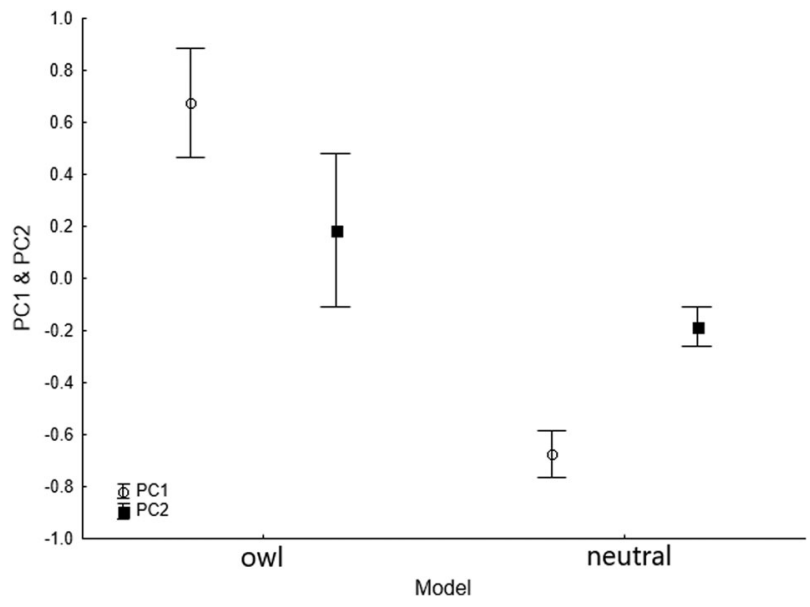

Figure 3. Principal components (PC1 and PC2) from the PCA based on acoustic and visual variables recorded for breeding Chilean Swallows in the presence of owl and neutral models. Trials for 8-d and 14-d nestlings were grouped. Dots and whiskers indicate mean time \pm se.

visual displays. Our preliminary analysis of the vocal repertoire of the Chilean Swallow identified a mobbing alarm call as part of the defensive repertoire. The results conform to our predictions only partially, for although Chilean Swallows performed appreciable antipredator responses (prediction a), there was no difference in defensive behaviours exhibited by adults rearing middle-aged or late nestlings, as we expected (prediction b).

In most aspects evaluated in this study, the antipredator responses observed for breeding Chilean Swallow adults were similar to those of Northern Hemisphere congeners (Shields 1984, Winkler 1991, 1992, 1994), which also display dives and circling flights around the predator. Moreover, dives and circling flights seem to be common in negative interactions because they were also used in aggressive conspecific interactions in the White-Rumped Swallow Tachycineta leucorrhoa (Wischhoff et al. 2018). The rapid response of other Chilean Swallows (possibly, pairs and their neighbours), who gathered within seconds, is also in line with behaviours recorded for swallows in other genera (e.g. Barn Swallow Hirundo rustica; Shields 1984). The number of Chilean Swallows involved in the mobbing events was smaller than for other congeners (Winkler 1994), probably due to the limited number of birds in our study colonies.

All vocalizations recorded were emitted when the swallows faced a threatening or non-threatening bird, and might, therefore, serve more than one function, which is usual in most birds (Marler 2004). Vocalization type A was the only type dominant in response to the owl model, while it was rarely given by swallows confronting the neutral model. An increase in the frequency of repetition of vocalization $\mathrm{A}$, combined with the visual displays, might act as a trigger to attract neighbouring conspecifics. Regarding the other vocalizations, the Chilean Swallow song type (named D) was more frequently emitted in the presence of the neutral model and needs to be further studied. Although not statistically significant, vocal types $\mathrm{B}$ and $\mathrm{C}$ were also more common in the presence of the predator, which might suggest an alarm or warning function.

Variation in nest defence has been linked to offspring age in many bird species (Tryjanowski \& Golawski 2004, Pavel 2006, Shew et al. 2016), including swallows. Northern Hemisphere Tree Swallows Tachycineta bicolor chased away threatening intruders increasingly as nestlings grew (Lombardo 1991). Also, Barn Swallows exposed to a predator model increased the intensity of mobbing from incubation towards the nestling stage (Shields 1984). However, Chilean Swallows did not show this pattern in our study. It is possible that Chilean Swallows invest as much energy in defending their nests from the beginning of the breeding attempt as they do later on, maintaining the level of antipredator behaviour throughout the entire nestling period. Southern Hemisphere Tachycineta swallows seem to have more dedicated parental care than Northern Hemisphere congeners, as shown by the higher feeding rates of White-Rumped Swallow, compared to the Tree Swallow (Bulit et al. 2014), and to the active role of Chilean Swallow males during incubation (Ospina et al. 2015).

In Northern Hemisphere swallows, Winkler (1992) found that males performed more intense displays than females, and Lombardo (1991) found that males chased intruders more often than did females. Unfortunately, we were unable to evaluate differences between sexes in defence behaviours of Chilean Swallows, as parental sex was not identified during the trials.

In conclusion, Chilean Swallow adults actively defended the nest surroundings from potential predators by displaying dives and circling flights and 
emitting loud mobbing alarm calls, apparently with the same intensity regardless of nestling age. Moreover, the immediate response of neighbouring swallows, resulting in group mobbing, was very common in our study system. This study provides preliminary clues on the functional value of the components of the vocal repertoire in the Chilean Swallow, which require further research. Further studies are needed to determine whether Chilean Swallows increase their reactiveness towards potential predators from the incubation to the chick-rearing stages. Larger samples are needed to compare each nestling stage, as these behavioural differences may be subtle. Also, identifying the sex of the parents and neighbouring swallows would be key to determine their individual role in nest defence and to study the social structure of mobbing groups.

\section{Acknowledgments}

We thank the Fortín Chacabuco TNC crew (G. Iglesias, G. Hulsegge, and N. Rodríguez), and the El Desafío owners and managers (L. Bachmann, R. Soto and T. Crespo). We also thank L. Chazarreta, A. Ortiz, G. Antolín and J. Hite for their help with setting up the nest-box colonies. We much appreciate the support from the Administración de Parques Nacionales in obtaining wooden planks, especially A. Moretti (Conservation Dept., Nahuel Huapi National Park) and A. Vereertbrügghen (Hotel Tronador). We thank two anonymous reviewers for their valuable comments. Research was conducted under permit 1523 to VO from Dirección Regional Patagonia Norte (DRPN, APN). All authors are members of the Consejo Nacional de Investigaciones Científicas y Técnicas (CONICET), Argentina. All authors declare that they have no potential conflict of interest.

\section{References}

Barash, D.P. 1975. Evolutionary aspects of parental behaviour: distraction behaviour of the Alpine Accentor. Wilson Bull. 87: 867-373.

Bulit, F., Barrionuevo, M. \& Massoni, V. 2014. Insights into life history theory: a brood size manipulation on a southern Hemisphere species, Tachycineta leucorrhoa, reveals a fast pace of life. J. Avian Biol. 45: 225-234.

Crawley, M.J. 2007. The $R$ Book. John Wiley \& Sons, Chichester.

Greig-Smith, P.W. 1980. Parental investment in nest defence by Stonechats (Saxicola torquata). Anim. Behav. 28: 604619.

Harvey, P.H. \& Greenwood, P.J. 1978. Anti- predator defence strategies: some evolutionary problems. In Krebs, J.R. \& Davies, N.B. (eds) Behavioural Ecology: An Evolutionary Approach, 129-151. Blackwell, Oxford.

Hogstad, O. 2005. Sex-differences in nest defence in Fieldfares Turdus pilaris in relation to their size and physical condition. Ibis 147: 375-380.
Ibarra, J.T., Altamirano, T.A., Martin, K., Vargas, F.H. \& Bonacic, C. 2014. Tree-cavity nesting of Austral PygmyOwls (Glaucidium nana) in Andean temperate forests of Southern Chile. J. Raptor Res. 48: 82-85.

Johnson, W.A. 1967. The Birds of Chile and Adjacent Regions of Argentina, Bolivia and Peru, Vol. 2. Platt Establecimientos Gráficos S. A., Buenos Aires.

Kaiser, H.F. 1958. The varimax criterion for analytic rotation in factor analysis. Psychometrika 23: 187-200.

Kontiainen, P., Pietiäinen, H., Huttunen, K., Karell, P., Kolunen, H. \& Brommer, J.E. 2009. Aggressive Ural owl mothers recruit more offspring. Behav. Ecol. 20: 789-796.

Liljesthrom, M. 2011. Biología reproductiva de la Golondrina Patagónica Tachycineta meyeni en Ushuaia, Tierra del Fuego. PhD Thesis, University of Buenos Aires, Argentina.

Lombardo, M.P. 1991. Sexual differences in parental effort during the nestling period in Tree Swallows (Tachycineta bicolor). Auk 108: 393-404.

Mahr, K., Riegler, G. \& Hoi, H. 2015. Parental risk management in relation to offspring defence: bad news for kids. Proc. R. Soc. Lond. B: Biol. Sci. 282: 20141670.

Marler, P. 2004. Bird calls: a cornucopia for communication. In Marler, P. \& Slabbekoorn, H. (eds) Nature's Music. The Science of Birdsong, 132-177. Elsevier Academic Press, London.

Massoni, V., Bulit, F. \& Reboreda, J.C. 2007. Breeding biology of the White-rumped Swallow Tachycineta leucorrhoa in Buenos Aires Province, Argentina. Ibis 149: 10-17.

Montgomerie, R.D. \& Weatherhead, P.J. 1988. Risks and rewards of nest defence by parent birds. Q. Rev. Biol. 63: 167-187.

Ospina, E., Cooper, C., Liljesthröm, M., Ardia, D. \& Winkler, D. 2015. Biparental nest-attendance in Chilean swallows (Tachycineta meyeni) breeding in Ushuaia, Argentina. Emu 115: 76-79.

Paruelo, J.M., Beltran, A., Jobbagy, E., Sala, O.E. \& Golluscio, R.A. 1998. The climate of Patagonia: general patterns and controls on biotic processes. Ecología Austral 8: $85-101$.

Pavel, V. 2006. When do altricial birds reach maximum of their brood defence intensity? J. Ethol. 24: 175-179.

Pinheiro, J., Bates, D., DebRoy, S. \& Sarkar, D. 2014. Linear and Nonlinear Mixed Effects Models. R package, version 3.

R Development Core Team. 2010. R: A Language and Environment for Statistical Computing. R Foundation for Statistical Computing, Vienna.

Redondo, T. \& Carranza, J. 1989. Offspring reproductive value and nest defence in the Magpie (Pica pica). Behav. Ecol. Sociobiol. 25: 369-378.

Ricklefs, R.E. 1969. An analysis of nesting mortality in birds. Smithson. Contrib. Zool. 9: 1-48.

Shew, J.J., van der Merwe, J., Schauber, E.M., Tallitsch, B.K. \& Nielsen, C.K. 2016. A classic question revisited in Red-Winged Blackbirds: disentangling confounding hypotheses surrounding parental investment theory and nest defence intensity. Behav. Ecol. Sociobiol. 70: 1843-1856.

Shields, W.M. 1984. Barn Swallow mobbing: self-defence, collateral kin defence, group defence, or parental care? Anim. Behav. 32: 132-148. 
Statsoft Inc. 2004. Statistica for Windows. V. 7. StatSoft Inc., Tulsa.

Svagelj, W.S., Trivellini, M. \& Quintana, F. 2012. Parental investment theory and nest defence by Imperial Shags: effects of offspring number, offspring age, laying date and parent sex. Ethology 118: 251-259.

Tryjanowski, P. \& Goławski, A. 2004. Sex differences in nest defence by the Red-Backed Shrike Lanius collurio: effects of offspring age, brood size, and stage of breeding season. J. Ethol. 22: 13-16.

Vrublevska, J., Krama, T., Rantala, M.J., Mierauskas, P., Freeberg, T.M. \& Krams, I.A. 2015. Personality and density affect nest defence and nest survival in the Great Tit. Acta Ethol. 18: 111-120.

Winkler, D.W. 1991. Parental investment decision rules in Tree swallows: parental defence, abandonment, and the so-called Concorde Fallacy. Behav. Ecol. 2: 133-142.
Winkler, D.W. 1992. Causes and consequences of variation in parental defence behaviour by Tree Swallows. Condor 94: 502-520.

Winkler, D.W. 1994. Anti-predator defence by neighbours as a responsive amplifier of parental defence in tree swallows. Anim. Behav. 47: 595-605.

Wischhoff, U., Marques-Santos, F., Manica, L.T., Roper, J.J. \& Rodrigues, M. 2018. Parenting styles in White-rumped swallows (Tachycineta leucorrhoa) show a trade-off between nest defence and chick feeding. Ethology. doi:10. 1111/eth.12770

Zanette, L.Y., White, A.F., Allen, M.C. \& Clinchy, M. 2011. Perceived predation risk reduces the number of offspring songbirds produce per year. Science 334: 1398-1401.

Zuur, A.F., Leno, E.N., Walker, N.J., Saveliev, A.A. \& Smith, G.M. 2009. Mixed Effects Models and Extensions in Ecology with $R$. Springer, New York. 